\title{
General Vacuum Electronics
}

\author{
(Invited Paper) \\ Jinjun Feng* $\cdot$ Xinghui Li $\cdot$ Jiannan $\mathrm{Hu} \cdot$ Jun Cai
}

\begin{abstract}
The electron devices in which electrons do not collide with other particles or in which the collision probability is very small in the transport process can be theoretically regarded as general vacuum electron devices. General vacuum electron devices include microfabricated vacuum nano-electronic devices, which can work in atmosphere, and some solid-state electron devices with nanoscale channel for electrons whose material characteristics are close to those of vacuum channels. Vacuum nano-electron devices (e.g., nanotriodes) are expected to be the fundamental elements for high-speed, radiation-resistant large-scale vacuum integrated circuits. The solid-state electron devices with spin semiconductor materials, multiferroics or topological crystal insulators are quite different from traditional semiconductor devices and are expected to operate under novel principles. Understanding vacuum electron devices from a microcosmic perspective and understanding solid-state electron devices from a vacuum perspective will promote a union of vacuum electronics and microelectronics, as well as the formation and development of general vacuum electronics.
\end{abstract}

Key Words: General Vacuum Electronics, Multiferroics, Spin Semiconductor Materials, Topological Crystal Insulators, Vacuum NanoElectron Devices.

\section{INTRODUCTION}

As a branch of electronics, vacuum electronics is the science of studying the generation and control of charged particles, as well as the interaction with matters and electromagnetic fields in vacuum or gas [1]. Materials and technologies adapted to the working environment are the technical basis of vacuum electronics. Vacuum electron devices use the principles of electrostatic and electromagnetic control, electron bunching exciting high-frequency field, high electric conductivity ability of gas discharge, scanning of electron beam to realize rectification, $\mathrm{RF}$ oscillation and amplification, detection, photography, display, frequency control and photoelectric conversion in electronic instruments and equipment. Nowadays, vacuum electronicsrepresented by high-frequency and high-power electron devices, including microwave, millimeter wave and terahertz power devices - is a very active research field.

In traditional vacuum electronics, a high vacuum environment with $10^{-7} \mathrm{~Pa}$ pressure is usually required to ensure the normal operation of devices. While with the rapid development of new nanomaterials, nanostructures and microfabrication technologies, vacuum can be viewed from another perspective, which is the essence of vacuum electronics: that is, the collision probability between the particles in the microstructure of the medium is quite small, or there is not any collision. In the case of nanoscale active regions of vacuum electron devices, high performance can be obtained with the significant reduction of strict requirements for vacuum.

With the advance of integration capability, semiconductor materials for solid-state electron devices are also developing very fast. The new materials are expected to construct sparse chan-

Manuscript received December 4, 2019. (ID No. 20191204-105J)

National Key Laboratory of Science and Technology on Vacuum Electronics, Beijing Vacuum Electronics Research Institute, Beijing, China.

"Corresponding Author: Jinjun Feng (e-mail: fengjinjun@tsinghua.org.cn)

This is an Open-Access article distributed under the terms of the Creative Commons Attribution Non-Commercial License (http://creativecommons.org/licenses/by-nc/4.0) which permits unrestricted non-commercial use, distribution, and reproduction in any medium, provided the original work is properly cited.

(c) Copyright The Korean Institute of Electromagnetic Engineering and Science. All Rights Reserved. 


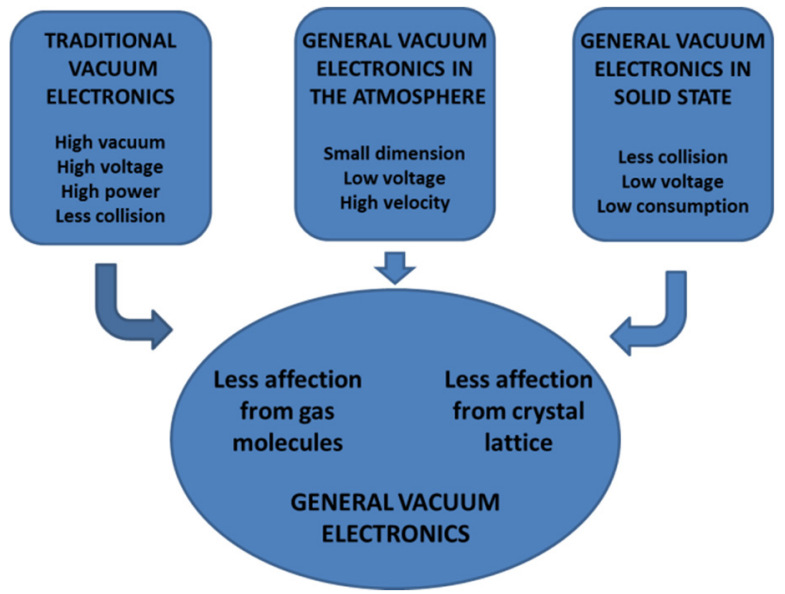

Fig. 1. Scientific connotation of general vacuum electronics.

nels in which electrons have high carrier mobility and long average free paths to realize fast, efficient and approximate ballistic transport of carriers in the channels with very short distances of nanometer scale.

With various vacuum degrees, solid-state electron devices or vacuum electron devices are of no matter, as long as the characteristic dimension of the channels for electrons inside the material is comparable to or less than the electron average free path; that is, as long as electrons do not collide with other particles, or the collision probability is very small in the transport process, the devices can be theoretically regarded as general vacuum electron devices. These devices will inherit the properties of vacuum devices. Understanding vacuum electron devices from a microcosmic perspective and understanding solid-state electron devices from a vacuum perspective will promote the integration of the two devices, as well as the formation and development of general vacuum electronics (Fig. 1).

\section{GENERAL VACUUM ELECTRONICS IN THE}

\section{ATMOSPHERE}

Vacuum electron devices are the active devices which can generate or amplify RF signals through the transmission of electrons or ions between electrodes and their interactions with electromagnetic field in vacuum. The main characteristics of vacuum electron devices are as follows: the electrons can be accelerated to very high velocities close to light speed in vacuum, so they have fast ON/OFF switch function and high kinetic energy; the electrons can work at very high voltage and current density by magnetic field confinement, so they can deliver high power RF output abstracted from the electron beams with high conversion efficiency.

Vacuum electron devices have obvious advantages in high $\mathrm{RF}$ power applications, but they encounter great difficulties in miniaturization, low power and complex function applications. This is because the size and precision of traditional vacuum electron devices are limited by high temperature thermionic cathode and vacuum sealing requirements, and it is difficult to achieve device miniaturization or system integration. The comparison of vacuum electron devices and solid-state electron devices show that the present vacuum electron devices with advantages in operation principle are seriously hindered by their fabrication and processing methods.

There are several major obstacles to the miniaturization, integration and realization of complex function of vacuum electron devices: mechanical manufacturing and manual assembly of discrete devices, thermal cathode power consumption and the integrating difficulty of heating components and the required high vacuum environment for device operation [2]. Two important technological breakthroughs in history have promoted the miniaturization process of vacuum electron devices: one is the emergence of microscale and nanoscale technologies of integrated circuits, including nanomaterials, nanostructures and nanofabrications that are used for field emission cathodes and some key fundamental components, such as all integrated vacuum diodes and triodes [3-5]; the field emission cathodes have made impressive progress in their properties, with high current density of $100 \mathrm{~A} / \mathrm{cm}^{2}$, and their applications in microwave tubes. The cold cathodes with field emitter arrays have been used in traveling wave tubes (TWTs) operating at $\mathrm{C}$ and $\mathrm{X} / \mathrm{Ku}$-band frequencies, offering coverage from $4 \mathrm{GHz}$ to $18 \mathrm{GHz}$. The $100 \mathrm{~W}$ C-band TWTs were operated at $5 \mathrm{GHz}$ and $121 \mathrm{~mA}$ beam current, and the up-to- $26 \mathrm{~W} \mathrm{X/Ku} \mathrm{TWTs} \mathrm{worked} \mathrm{at} 10$ $\mathrm{GHz}$ and $80 \mathrm{~mA}$ beam current. The tests demonstrated over 1,000 hours operating time over four prototypes. There are hopes to operate towards millimeter wave frequency band with $10 \mathrm{~A} / \mathrm{cm}^{2}$ current density, which is the parameter regime not possible for long life thermionic cathodes [6]. Microelectromechanical systems (MEMS) technology, a high aspect ratio (HAR) micro-fabrication process, is used in the micro-fabrication of slow-wave structures, with dimensions around several tens of microns for high-frequency vacuum devices, also called high-frequency integrated vacuum electronics that promote the terahertz vacuum devices, the miniaturization and integration of devices [7, 8]. The MEMS technologies include UV-LIGA for copper parts fabrication and deep reactive ion etching (DRIE) for silicon etching with metal thin-film coating, which have been used in the terahertz vacuum power devices from 220 $\mathrm{GHz}$ up to $1 \mathrm{THz}$ with power from tens of milliwatts to over one hundred watts.

In recent years, the rapid development of micro-fabrication technology has reduced the characteristic dimensions of the vacuum devices to microscale and nanoscale, which opens the way for the realization of micro/nano-vacuum devices. In 2012, the NASA Ames Research Center proposed the concept of an 
air channel transistor with insulating gate structure by referring to field-effect transistors (FETs) and transverse vacuum triodes [9]. The insulating layer between the emitter and gate eliminates the possibility of gate electron interception, and the very small distance ensures that the atmospheric environment between emitter and collector can be regarded as a vacuum channel, which is similar to the semiconductor electron transmission channel in a traditional transistor. The concept of an air channel transistor achieves the perfect combination of vacuum operation principles and device miniaturization. The distance between the electrodes is expected to be about $10 \mathrm{~nm}$. The preliminary experimental device is realized by projection lithography and photo resistant plasma-ashing technology. The collector is $120 \mathrm{~nm}$ away from the emitter, the working voltage is less than $10 \mathrm{~V}$ and the corresponding cut-off frequency is $0.46 \mathrm{THz}$. As a preliminary attempt, the device still needs to be improved and optimized in the design and fabrication process, including improvements to emitter material, film thickness, electrode shape and position, so as to reduce the working voltage to about $1 \mathrm{~V}$ and achieve better device performance.

Also in 2012, the University of Pittsburgh proposed the design of a vacuum channel metal oxide semiconductor (MOS) FET, which has a vertical field emission structure [10]. Unlike the traditional Spindt-type microtriode, the new device uses a thin film edge emitter other than a cone field emitter, and the distance between electrodes is controlled through the adjustment of the insulator thin-film thickness other than the microfabricated slot. The distance between emitter and gate is precisely limited to $20-30 \mathrm{~nm}$, the working voltage of the device is as low as $0.5 \mathrm{~V}$, and the current ratio of ON/OFF state is over 500 at $2 \mathrm{~V}$. In atmospheric environment, the thin-film edge emission current density is about $10^{5} \mathrm{~A} / \mathrm{cm}^{2}$ under the assumption that all electrons are emitted from a thin film with $1 \mathrm{~nm}$ thickness. The transconductance of the structure is $20 \mathrm{nS} / \mu \mathrm{m}$ but is expected to be $2 \mu \mathrm{S} / \mu \mathrm{m}$ by increasing the anode electron collecting efficiency and optimizing the device structure.

Recently, a nanoscale edge emission vacuum channel triode was realized in Beijing Vacuum Electronics Research Institute (BVERI), China. The structure and the characteristic dimension of the device are similar to those of the device from the
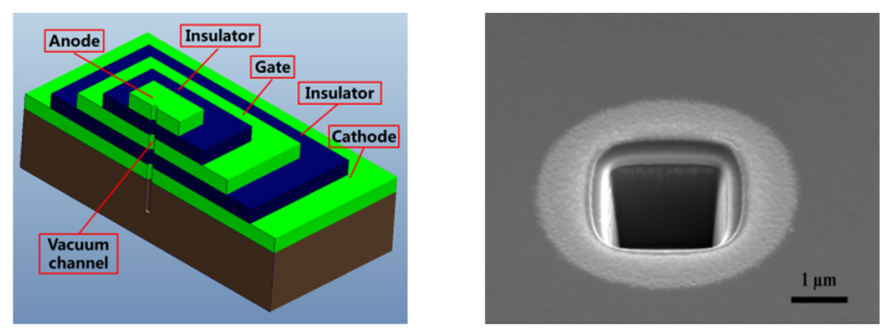

Fig. 2. Section diagram of nanoscale edge emission vacuum channel triode; and SEM image of a focused ion beam (FIB) etched vacuum channel (BVERI).
University of Pittsburgh, but it has a molybdenum layer as the cathode instead of n-type silicon (Fig. 2). The turn-on voltage of the device is as low as several volts. The diode characteristics test between cathode and anode shows that the device can maintain working in a large range of vacuum environments. Although there are still sample destruction and failures in abrupt vacuum changing, most devices can work with a stable emission current from $10^{-7} \mathrm{~Pa}$ up to atmosphere (Fig. 3).

Researchers believe that the new vacuum nano-electron devices may replace the existing MOS transistors to achieve highspeed switching and signal amplification, and the vacuum integrated circuits will also compete strongly with solid-state integrated circuits [11-13]. Especially in space exploration field, vacuum nano-electron devices and vacuum integrated circuits have particular advantages. Due to the small size, light weight, high integration, high efficiency and no preheating warm-up time, solid-state electron devices have obvious advantages over vacuum electron devices in normal applications and are suitable for phase array systems and aerospace engineering. However, failure analysis in the manned spaceflight field in recent decades shows that the accumulation of space radiated particles can damage nanoscale solid-state electron devices, resulting in the decrease of $\mathrm{ON}$-state current, the increase of leakage current or even the logic confusion of the circuits. Although the instantaneous and cumulative damage of this space environment can be

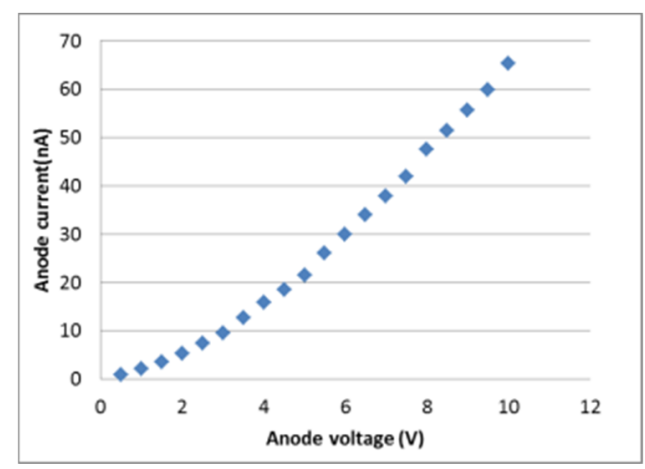

(a)

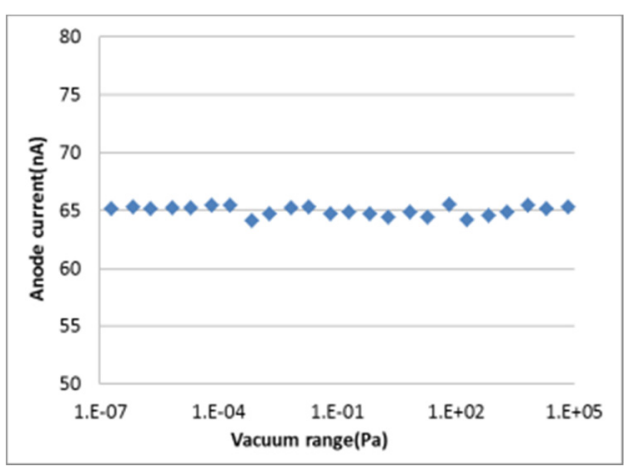

(b)

Fig. 3. (a) I-V characteristics between cathode and anode and (b) emission stability test with different vacuum at anode bias of $10 \mathrm{~V}$. 
resisted and the data can be restored by system backup, there is no way to deal with some catastrophic damages. This kind of electronic failure of solid-state electron devices seriously hinders the progress of space exploration projects and even leads to the failure of missions. The vacuum channel nanostructure has basic functions similar to those of the traditional vacuum tube. Because both the device emitter and collector are in a vacuum state and there are not any properties from solid material, it will therefore not be damaged by space radiation.

The novel vacuum nano-electron devices can be manufactured by existing silicon-based process technologies to achieve good electrical properties with radiation-resistance and extreme temperature resistance, while further performance improvements can also be expected by introducing advanced materials such as silicon carbide and graphene, etc. Many research institutions have paid attention to the devices and technologies and have followed up to study the device principles, materials, and processes.

Although these two devices are quite different in structures, they have two revolutionary common features: one is that the characteristic dimensions of the devices are all in nanometer scale and the other is that the operating voltages of the devices are all less than $10 \mathrm{~V}$. Nanoscale characteristic dimension is neither the simple size decline nor the simple name transition from microdevice to nanodevice. Since the microfabricated distance between electrodes is close to or less than the molecules' average free path in atmospheric state (about $60 \mathrm{~nm}$ ), it means that even in atmospheric environment, electrons can transport between electrodes almost without colliding with gas molecules. In other word, the nanoscale atmospheric electron channel can be "regarded as vacuum". Similarly, the working voltage decrease from several tens of volts or above $100 \mathrm{~V}$ to less than $10 \mathrm{~V}$ is not a simple digital decline. Because the $10 \mathrm{~V}$ keeps electrons' energy less than the air molecules' first ionization energy in their whole working process, it means that even if the electrons collide with the gas molecules, the gas will not be ionized. In other words, the very low working voltage nanoscale vacuum devices can "exclude ionization".

This kind of micro/nano-vacuum device based on the most advanced microfabrication technology is gradually breaking through the traditional vacuum electron device mode and bringing it into a new research field. The mode of normal operation in atmospheric environment based on the principle of traditional vacuum devices can be called general vacuum electronics in the atmosphere. The theoretical essence of it is "regarded as vacuum" and "excluding ionization"; the core devices are vacuum micro/nano-electron devices represented by vacuum nanodiodes and nanotriodes; and the ultimate goal is to achieve integrated power sources or high-speed, radiation-resistant largescale vacuum integrated circuits.

\section{GENERAL VACUUM ELECTRONICS IN SOLID STATE}

In 1965, Moore's law proposed that the number of components that can be accommodated on the integrated circuit (IC) doubles every 18 to 24 months, and the performance doubles accordingly $[14,15]$. The improvement of IC integration on chips mainly depends on the improvement of microfabrication technology, represented by lithography and etching technology. In order to shrink the characteristic size that is normally indicated by the line width of the circuits, optical lithography with different wavelength light sources, as well as non-optical lithography with shorter equivalent wavelength (including electron beam lithography, focused ion beam lithography and X-ray lithography), were developed. Meanwhile, many other etching methods, e.g., wet etching, reactive ion etching, ion milling and high ion density etching, as well as electron synchronous cyclotron resonance and inductively coupled plasma etching techniques, have also been developed. The rapid development of other related technologies, such as ultra-thin film growth and deposition, large beam current and low-energy ion implantation for precise doping, shallow junction ohmic contact and multilayer wiring for increasing the components' density, have also greatly improved microfabrication technology. The typical processing size gradually decreased from about 50 microns in the 1960s to less than 0.2 microns in 2000, and the nanometer scale has been further developed recent years. However, according to the International Technology Roadmap for Semiconductors (ITRS), silicon complementary metal oxide semiconductor (CMOS) transistors will reach their physical limits in 2020 [16, 17]. In 2015, Intel announced that it was abandoning silicon in 7 nanometer technology nodes and looking for other materials for the channel to develop advanced integrated circuit technology.

In addition to microfabrication technologies, solid-state devices are limited by the physics principle. In essence, carriers inside a solid can hardly avoid lattice and impurity scattering, leading to failure of ballistic transport. Thus, the structure and operating principles of solid-state devices restrict the development of their output power and frequency. As semiconductor devices are near their material limits and as the shrinkage of microfabrication feature size becomes more difficult, significant improvements can hardly be made to performance.

Therefore, how to develop new materials and explore novel principles based on existing manufacturing technologies to obtain advanced solid-state electronic devices with characteristics of fewer particle collisions and lower energy consumption is quite a critical issue. This would help improve the existing solidstate devices. More importantly, the field of traditional vacuum devices will also expand, since the channels of this kind of device are expected to have characteristics similar to those of the vacu- 
um channel. Thus, the related field can be called "generalized vacuum electronics in solids", although microelectronic technology has been adopted. In particular, spin semiconductor materials, multiferroics and topological crystal insulators can be considered as the material of such generalized vacuum electronics.

\section{Spintronic Devices}

As a research direction of novel electronic devices, spintronics is a discipline that studies and utilizes the electron spin degrees of freedom to combine or replace charge degrees of freedom [18]. Its main research goal is to replace traditional transistors and realize spin transistors.

Spintronic devices have good compatibility with the materials and microfabrication technology of traditional semiconductor electronic devices that are based on charge mechanisms. In the past 20 years, the research of semiconductor spintronics has made great progress in device design, experimental technology and theoretical methods. In 2009, the Datta-Das spin-FET was realized in the non-local spin valve structure of InAs quantum well [19]. The gate voltage is used to control the spatial precession of spin during the transport process, and the switch function was realized. Moreover, the structures of unipolar spin transistor $[20,21]$ and hot-electron spin transistor $[22,23]$ are also possible candidates of spin transistor.

In traditional semiconductor devices, the charges' characteristics of carriers rather than electron spin is used to transmit information. The orientation of the electron spin is random, and they will not, then, carry any information. In contrast, spintronic devices take advantage of electron spin to process information by manipulating the spins. Since the spin flip needs lower energy and shorter time compared to the carrier channel switch, lower energy consumption and higher speed can be achieved. From this aspect, spintronic devices can be considered as one kind of generalized vacuum electronic devices.

New materials such as multiferroics, topological insulators and topological crystal insulators have been discovered and studied recently. These materials exhibit unique physical properties related to the spin whose electron transport properties are similar to those of the vacuum, which are the properties of electron ballistic motion.

\section{Novel Interface of Oxides}

In 2004, it was reported in Nature magazine that a quasitwo-dimensional electron gas was found at the insulating oxide $\mathrm{LaAlO}_{3} / \mathrm{SrTiO}_{3}$ heterostructure interface [24] whose mobility was a very high reaching $10,000 \mathrm{~cm}^{2} \mathrm{~V}^{-1} \mathrm{~s}^{-1}$ at low temperature and whose carrier density was an order of magnitude higher than that of III-V semiconductor heterostructure. Based on this, the possibility of using this quasi-two-dimensional electron gas to build a FET with high electron mobility was explored in
2006 [25]. It was found that when the thickness of $\mathrm{LaAlO}_{3}$ on the 1-mm-thick $\mathrm{SrTiO}_{3}$ substrate is larger than 3 unit cells, the electrical conductivity of the interface would significantly increase by two orders of magnitude. In order to explore the influence of gate voltage on carrier transport characteristics of a 3unit-cells-thick sample, the curve of voltage and current was tested in the experiment, and the influence of temperature on the sheet resistance was studied. The results showed that the conductivity of two-dimensional electron gas could be changed by seven orders of magnitude by tuning gate voltage, indicating that oxides have great potential in establishing a FET. This would also provide an effective way to build channels similar to vacuum.

It is considered that the discontinuity of polarity accounts for the existence of two-dimensional electron gas. In this way, a reversal of polarity would introduce greater polarity discontinuities; thus, theoretically, it is also a possible structure to realize two-dimensional conductivity. In 2012, a variety of surface measurement methods were adopted to study the conductivity of polarity reversal interface of $\mathrm{HoMnO}_{3}$ ferroelectric materials (domain wall) [26]. The results showed that the domain walls are conductive, and it is necessary to further explore such structures.

This research shows that when using the polarity discontinuity of the heterostructure with ferroelectric material, the conductive channel with high conductivity and high carrier mobility can be constructed. Compared with the traditional semiconductor channel, they have greater advantages in conductivity and are closer to the electron transmission in vacuum with much less lattice scattering.

\section{Topological Insulator and Topological Crystal Insulator}

In addition to the oxide materials mentioned above, topological insulators are also of wide concern to researchers, as they would result in the realization of electronic devices with low power consumption [27, 28]. Based on the concept of topological insulators, the novel properties such as quantum spin Hall Effect, quantum anomalous Hall effect and Majorana fermion are expected to help realize the next generation of chips for computers.

With the progress on topological insulators, another new material system with topological properties-topological crystal insulators-was proposed in 2011 [29]. Topological crystal insulators have more abundant physical properties, especially the quantum spin hall effect, which can be controlled by electric field.

In 2014, the concept of a three-dimensional topological crystal insulator was extended to two-dimensional, and a new topological phase of a two-dimensional insulator protected by mirror symmetry was proposed [30]. It was proved that this new phase 
can be realized in SnTe (001) and PbxSn1-xSe (Te) (001) thin films. In this material, the band of bulk has band gap, but the edge state protected by (001) mirror symmetry is with spin filter property. More importantly, the mirror symmetry of the system can be destroyed by using the electric field perpendicular to the thin film, thereby creating an electric field-tuned band gap of the edge of the film, which is called edge state. Taking advantage of this singular property, the conduction of the film edge can be accomplished with no energy consumption when the band gap is tuned to zero; thus, a brand-new type of transistor, called a topological transistor, was proposed.

Transistors are the essential elements of all modern electronic systems. Traditional transistors are based on the $\mathrm{P}-\mathrm{N}$ junctions, using $\mathrm{P}$ - and $\mathrm{N}$-type semiconductors, while the $\mathrm{ON} / \mathrm{OFF}$ states are realized through the recombination of electrons and holes. During these processes, a large amount of heat is generated, which is a waste of energy and affects the integration density of the electronic devices. In addition, the velocities of electrons and holes within semiconductor materials are quite limited, which causes the applications of traditional transistors to be limited, especially in much higher frequency regions.

The principle of a topological transistor is completely different from that of a traditional transistor. In a topological transistor, charge transport and spin transport are strongly entangled, and high switching speed and low energy consumption can be achieved. Therefore, this is also a new way to build conductive channels with vacuum characteristics, which is very important in spintronics and in the information superhighway.

However, in topological transistors, the carrier transport and spin transport are strongly entangled together, and they can be controlled by the external electric field. The principle of the topological transistor is completely different from that of traditional transistors, and their ON/OFF states do not rely on the combination of $\mathrm{N}$-type electrons and $\mathrm{P}$-type holes. Instead, they rely on the tunable band gap of the edge state, and thereby the tunable conduction of the film edge. Therefore, rather high switch speed and low energy cost can be realized. Thus, this is also a completely new way to construct a conductive channel with characteristics similar to those of vacuum, and topological transistors will play great roles in the next generation of the information highway and topological quantum computing.

\section{CONCLUSION}

Several electron devices, including microfabricated vacuum nano-electron devices, solid-state electron devices with spin semiconductor materials and multiferroics or topological crystal insulators are introduced. Each distinct device has similar electron characteristics and can be defined as general vacuum electron devices. The definition promotes the formation of general vacuum electronics and opens up a new research area.

\section{REFERENCES}

[1] F. Liao, Z. Sun, and T. Yan, Vacuum Electronics. Beijing, China: National Defense Industry Press, 2008.

[2] K. R. Shoulders, "Microelectronics using electron-beamactivated machining techniques," Advances in Computers, vol. 2, pp. 135-293, 1961.

[3] C. A. Spindt and K. R. Shoulders, "Research in micron-size field-emission tubes," in Proceedings of the 8th Conference on Tube Techniques, New York, NY, 1966, pp. 143-145.

[4] C. A. Spindt, I. Brodie, L. Humphrey, and E. R. Westerberg, "Physical properties of thin-film field emission cathodes with molybdenum cones," Journal of Applied Physics, vol. 47, no. 12 , pp. 5248-5263, 1976.

[5] C. A. Spindt, "Method of fabricating a funnel-shaped miniature electrode for use as a field ionization source," US Patent 4141405 , Feb 27,1979.

[6] D. R. Whaley, C. M. Armstrong, C. E. Holland, C. A. Spindt, and P. R. Schwoebel, "Cold cathode based microwave devices for current and future systems," in Proceedings of the 31th International Vacuum Nanoelectronics Conference, Kyoto,Japan, 2018, pp. 64-65.

[7] J. Feng, J. Cai, Y. Hu, X. Wu, Y. Du, J. Liu, P. Pan, and H. Li, "Development of W-band folded waveguide pulsed TWTs," IEEE Transactions on Electron Devices, vol. 61, no. 6, pp. 1721-1725, 2014.

[8] Y. Du, J. Cai, P. Pan, R. Dong, X. Zhang, S. Liu, X. Wu, and J. Feng, "Experimental Investigation of an ultrawide bandwidth W-band pulsed travelling wave tube with microfabricated folded waveguide circuits," IEEE Transactions on Plasma Science, vol. 47, no. 1,pp. 219-224, 2019.

[9] J. W. Han, J. S. Oh, and M. Meyyappan, "Vacuum nanoelectronics: back to the future? - Gate insulated nanoscale vacuum channel transistor," Applied Physics Letters, vol. 100, article no. 213505, 2012.

[10] S. Srisonphan, Y. S. Jung, and H. K. Kim, "Metal-oxidesemiconductor field-effect transistor with a vacuum channel," Nature Nanotechnology, vol. 7, no. 8, pp. 504-508, 2012.

[11] University of Pittsburgh, "Nanoscientists suggest use of vacuums to overcome limits of conventional silicon-based semiconductor electronics," 2012; https://phys.org/news/ 2012-07-nanoscientists-vacuums-limits-conventional-silicon-based.html.

[12] B. R. Stoner and J. T. Glass, "Nanoelectronics: nothing is like a vacuum," Nature Nanotechnology, vol. 7, no. 8, pp. 485-487, 2012.

[13] J. Cartwright, "Return of the Vacuum Tube," 2012; https:// www.sciencemag.org/news/2012/05/return-vacuum-tube. 
[14] F. Meier and B. P. Zakharchenya, Optical Orientation. Amsterdam: North-Holland, 1984.

[15] S. D. Sarma, J. Fabian, X. Hu, and I. Zutic, "Spin electronics and spin computation," Solid State Communications, vol. 119, no. 4-5, pp. 207-215, 2001.

[16] J. F. Gregg, I. Petej, E. Jouguelet, and C. Dennis, "Spin electronics: a review," Journal of Physics D: Applied Physics, vol. 35, no. 18, pp. R121-R155, 2002.

[17] D. D. Awschalom, D. Loss, and N. Samarth, Semiconductor Spintronics and Quantum Computation. Berlin: Springer, 2002.

[18] I. Zutic, J. Fabian, and S. D. Sarma, "Spintronics: fundamentals and applications," Reviews of Modern Physics, vol. 76, no. 2, pp. 323-410, 2004.

[19] H. C. Koo, J. H. Kwon, J. Eom, J. Chang, S. H. Han, and M. Johnson, "Control of spin precession in a spin-injected field effect transistor," Science, vol. 325, no. 5947, pp. 1515-1518, 2009.

[20] M. E. Flatte and G. Vignale, "Unipolar spin diodes and transistors," Applied Physics Letters, vol. 78, no. 9, pp. 1273$1275,2001$.

[21] M. E. Flatte and G. Vignale, "Heterostructure unipolar spin transistors," Journal of Applied Physics, vol. 97, article no. 104508, 2015.

[22] I. Appelbaum, B. Huang, and D. J. Monsma, "Electronic measurement and control of spin transport in silicon," Nature, vol. 447, no. 7142, pp. 295-298, 2007.

\section{Jinjun Feng (M'94-SM'06)}

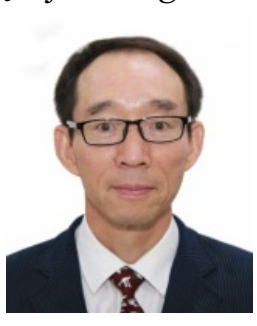

received the B.S. degree from the Department of Electronic Engineering, Tsinghua University, Beijing, China, in 1988 and the M.Sc. and Ph.D. degrees from Beijing Vacuum Electronics Research Institute (BVERI), Beijing, in 1990 and 2001, respectively. Since 1990, he has been with BVERI, and has been the Deputy Director of the National Key Laboratory of Science and Technology on Vacuum Electronics since 2004, and the Vice Chief-Engineer of BVERI since 2010 and the Vice Director-General since 2016, where his research focused on vacuum electronic devices including space TWT, millimeter-wave TWT, Terahertz vacuum sources using micro-fabrication, gyrotrons and cesium tubes etc. He has authored and co-authored over 300 papers in conferences and journals. Dr. Feng is currently a member of IEEE EDS Vacuum Electronics Technical Committee, Chairman of IEEE China Council, Fellow of Chinese Institute of Electronics (CIE) and the Fellow of the IET, UK. He got the John R Pierce Award of Excellence in Vacuum Electronics in 2019.
[23] B. Huang, D. J. Monsma, and I. Appelbaum, "Experimental realization of a silicon spin field-effect transistor," Applied Physics Letters, vol. 91, article no. 072501, 2007.

[24] A. Ohtomo and H. Y. Hwang, "A high-mobility electron gas at the $\mathrm{LaAlO} 3 / \mathrm{SrTiO}_{3}$ heterointerface," Nature, vol. 427, no. 6973, pp. 423-426, 2004.

[25] S. Thiel, G. Hammerl, A. Schmehl, C. W. Schneider, and J. Mannhart, "Tunable quasi-two-dimensional electron gases in oxide heterostructures," Science, vol. 313, no. 5795, pp. 1942-1945, 2006.

[26] W. Wu, Y. Horibe, N. Lee, S. W. Cheong, and J. R. Guest, "Conduction of topologically protected charged ferroelectric domain walls," Physical Review Letters, vol. 108, no. 7, article no. 077203, 2012.

[27] C. L. Kane and E. J. Mele, "Z2 topological order and the quantum spin Hall effect," Physical Review Letters, vol. 95, article no. 146802, 2005.

[28] X. L. Qi and S. C. Zhang, "Topological insulators and superconductors," Reviews of Modern Physics, vol. 83, no. 4, pp. 1057-1110, 2011.

[29] L. Fu, "Topological crystalline insulators," Physics Review Letters, vol. 106, article no. 106802, 2011.

[30] J. Liu, T. H. Hsieh, P. Wei, W. Duan, J. Moodera, and L. Fu, "Spin-filtered edge states with an electrically tunable gap in a two-dimensional topological crystalline insulator," Nature Materials, vol. 13, no. 2, pp. 178-183, 2014.

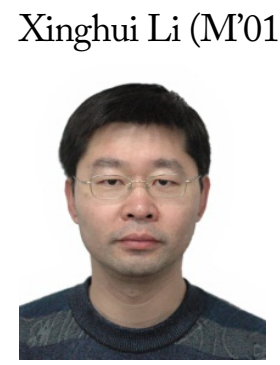

received the B.S. degree in Physical Electronics from the Department of Electronic Engineering, Xi'an Jiaotong University, Shaanxi, China, in 1993 and the M.Sc. and Ph.D. degrees in Physical Electronics from Beijing Vacuum Electronics Research Institute, Beijing, in 2001 and 2005, respectively. Since 1993, he has been with National Key Laboratory of Science and Technology on Vacuum Electronics, BVE$\mathrm{RI}$, where his research focused on vacuum micro/nano-electronic devices, field emission cathodes and microfabrication, etc. He has authored and coauthored over 100 papers in domestic/international conferences and journals. 
Jiannan $\mathrm{Hu}$

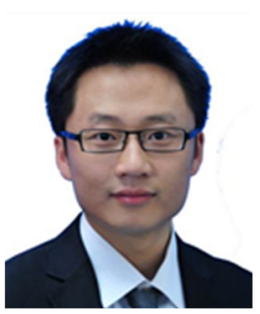

received the B.S. degree in physics and optoelectronics from Department of Physics and Department of Optoelectronic Information Science and Technology, Harbin Institute of Technology, Harbin, Heilongjiang, China, in 2007. After working as a research assistant focusing on growth of III-nitride semiconductor epilayers, he received the Ph.D. degree in physical electronics and optoelectronics from Department of Electronics Engineering, Tsinghua University, Beijing, China, in 2014. Since 2014, he has been with National Key Laboratory of Science and Technology on Vacuum Electronics, BVERI, where his research is mainly on growth of single crystalline diamond films.
Jun Cai (M’09)

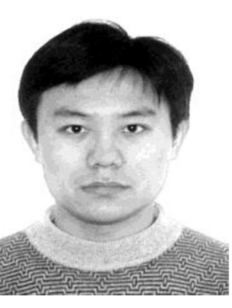

was born in Tangshan, Hebei, China, in 1978. He received the Ph.D. degree in radio physical from the Department of Information Science and Engineering, Shandong University, Jinan, China, in 2006. Since 2006, he has been with the National Key Laboratory of Science and Technology on Vacuum Electronics, Beijing Vacuum Electronics Research Institute, Beijing, China, where is involved in vacuum electronic devices, including W-band TWTs and terahertz vacuum electronic devices. 